\title{
ON NILPOTENCY IN THE ADO-HARISH-CHANDRA THEOREM ON LIE ALGEBRA REPRESENTATIONS
}

\author{
RICHARD E. BLOCK
}

\begin{abstract}
Let $L$ be a finite-dimensional Lie algebra, over an arbitrary field, and regard $L$ as embedded in its enveloping algebra $U L$.

THEOREM. If $K$ is an ideal of $L$ and $K$ is nilpotent of class $q$, then for any $r$ there exists a finite-dimensional representation $\rho$ of $L$ which vanishes on all products (in $U L)$ of $\geq q r+1$ elements of $K$ and is faithful on the subspace of $U L$ spanned by all products of $\leq r$ elements of $L$.

This result sharpens (with respect to nilpotency) the Ado-Iwasawa theorem on the existence of faithful representations and the Harish-Chandra theorem on the existence of representations separating points of $U L$.
\end{abstract}

This paper gives a sharpened (with respect to nilpotency) form of two classical theorems: Ado's theorem on the existence of a faithful representation of a Lie algebra, and the related theorem of Harish-Chandra on the existence of a representation not annihilating a given element of the universal enveloping algebra. Our results are valid both at characteristic 0 and at prime characteristic, although our proofs are different for the two cases.

Throughout the paper $L$ denotes a finite-dimensional Lie algebra, regarded as embedded in its enveloping algebra $U L$, and $N$ denotes the largest nilpotent ideal of $L$. Representation of $L$ will always mean finite-dimensional representation and, when convenient, the same notation will be used for a representation of $L$ and for the corresponding extension to a representation of $U L$.

We recall that Ado's theorem has a shorter version and a more precise version which are, respectively, as follows. ${ }^{1}$

THEOREM A. L has a faithful representation.

THEOREM B. L has a faithful representation $\rho$ which is nilpotent on $N$ (that is, $\rho(x)$ is nilpotent for all $x \in N)$.

By Engel's theorem, the nilpotency of $\rho$ on $N$ is equivalent to the existence of an $m>0$ such that $\rho\left(x_{1} \cdots x_{m}\right)=0$ for all $x_{1}, \ldots, x_{m} \in N$. In this paper we shall show how small a value of $m$ can be attained. In fact we shall prove the following

Received by the editors May 17, 1985 and, in revised form, October 22, 1985.

1980 Mathematics Subject Classification (1985 Revision). Primary 17B35, 17B10, 17B30, 17 B50.

Key words and phrases. Lie algebra, enveloping algebra, nilpotent ideal, nilpotency class, faithful representation.

Research supported in part by National Science Foundation Grant DMS-8201828 and DMS8502799.

${ }^{1}$ At characteristic 0, these results are known as Ado's theorem; E. Cartan, Hochschild, Iwasawa, Harish-Chandra, Zassenhaus, and Hochschild and Mostow also contributed proofs of Theorem A or $\mathrm{B}$ in this case. At prime characteristic, Theorem A is a result of Iwasawa, with a short proof having been given by Jacobson (see Chapter 6, "The theorem of Ado-Iwasawa," of [Ja2].) The prime characteristic version of Theorem B is covered by Hochschild [Ho2]. 
result (recall that the nilpotency class of a nilpotent Lie algebra $K$ is the smallest $q$ such that all bracket products of $q+1$ elements of $K$ are 0 , or equivalently, such that $\left.\left(\operatorname{ad}_{K} K\right)^{q}=0\right)$.

THEOREM 1. Suppose $K$ is a nilpotent ideal (of $L$ ) of nilpotency class $q$. Then there exists a faithful representation $\rho$ of $L$ which satisfies $\rho\left(x_{1} \cdots x_{q+1}\right)=0$ for all $x_{1}, \ldots, x_{q+1} \in K$.

Here the number $q+1$ of factors is the best possible, since if all products of $q$ elements of $\rho(K)$ are zero, then also all bracket products of $q$ elements of $\rho(K)$ are zero, and so by faithfulness $K$ has class $<q$, a contradiction. The result of Theorem 1 appears to be new even for $K=N$.

We next recall a result of Harish-Chandra, which is the main theorem of $[\mathbf{H a}]{ }^{2}$

THEOREM C. If $0 \neq u \in U L$ then there exists a representation $\rho$ of $L$ such that $\rho(u) \neq 0$.

For $i \geq 0$ let $(U L)_{i}$ denote the subspace of $U L$ spanned by all products of $\leq i$ elements of $U L$; thus $\left\{(U L)_{i}\right\}_{i \geq 0}$ is the canonical ascending filtration of $U L$. It is pointed out in [Ha] that the statement of Theorem $\mathrm{C}$ (whose proof uses Theorem A) in fact implies Theorem $\mathrm{A}$. We note that the same argument implies the following formal strengthening of Theorem $\mathrm{C}$.

THEOREM D. For any $r \geq 0$ there exists a representation of $L$ which is faithful on $(U L)_{r}$.

As our main result, we shall prove the following theorem, which extends all of the above and is valid at arbitrary characteristic.

THEOREM 2. Suppose $K$ is a nilpotent ideal (of $L$ ) of nilpotency class $q$. For any $r \geq 0$ there exists a representation $\rho$ of $L$ which is faithful on $(U L)_{r}$ and satisfies $\rho\left(x_{1} \cdots x_{q r+1}\right)=0$ for all $x_{1}, \ldots, x_{q r+1} \in K$.

Again the number $q r+1$ of factors is the best possible, since if $x$ is a nonzero bracket product of $q$ elements of $K$, then $0 \neq \rho\left(x^{r}\right) \in \rho(K)^{q r}$.

We shall proceed to the proof, using the following notation: $K$ denotes a nilpotent ideal (of $L$ ) of nilpotency class $q$; we may assume that $K \neq 0$. The terms of the lower central series of $K$ will be denoted by $K_{i}$, where $K_{1}=K$ and $K_{i+1}=\left[K, K_{i}\right]$; thus $K_{q+1}=0$ and $K_{q} \neq 0$. Also, for each $i, K^{i}$ will denote the span in $U L$ of $K K \cdots K$ ( $i$ times). Note that $K_{i} \subseteq K^{i}$. We define an integer-valued function $w$ on $K-\{0\}$ by $w(x)=n$ if $x \in K_{n}-K_{n+1}$. We take a basis $\left\{x_{1}, \ldots, x_{s}\right\}$ of $K$ which is compatible with the lower central series of $K$, that is, for each $i=1, \ldots, q$ there is an $l_{i} \in\{1, \ldots, s\}$ such that $\left\{x_{k} \mid l_{i} \leq k \leq s\right\}$ is a basis of $K_{i}$. Let $S_{k}$ denote the span of

$$
\left\{x_{j_{i}} \cdots x_{j_{n}} \mid n \geq 1,1 \leq j_{1} \leq \cdots \leq j_{n} \leq s, \sum_{i=1}^{n} w\left(x_{j_{i}}\right)=k\right\} .
$$

\footnotetext{
${ }^{2}$ Expositions of the theorem are also given in [Di] and in exercises in [Bo, Ja2]. Again, this theorem was only given at characteristic 0 , but its validity at prime characteristic follows from a modification of the proof (or extension) of the Ado-Iwasawa theorem by Jacobson [Ja, Ja2] or Hochschild [Ho2]. W. Michaelis has announced a coalgebra proof of the Harish-Chandra theorem at arbitrary characteristic.
} 
LEMMA 1. For any $k \geq 1$,

$$
\sum_{j \geq k} K^{j}=\sum_{j \geq k} S_{j}
$$

ProOF. Since $K_{i} \subseteq K^{i}, S_{k} \subseteq K^{k}$. In the process of straightening a monomial in $x_{1}, \ldots, x_{s}$ to express it as a linear combination of standard monomials, each straightening gives a linear combination of terms $x_{m_{1}} \cdots x_{m_{t}}$ for which $\sum_{i=1}^{t} w\left(x_{m_{i}}\right)$ has at least the previous value, since $\left[K_{i}, K_{j}\right] \subseteq K_{i+j}$. Hence $K^{j} \subseteq \sum_{j \leq l} S_{l}$.

We now prove Theorem 1 under the assumption that the characteristic is 0 . Let $z L$ denote the center of $L$. Then $K+z L$ is also nilpotent of class $q$, and so without loss of generality we may assume that $z L \subseteq K$. Let $I$ denote the ideal of $U K$ generated by $K^{q+1}$. Then $(U K) / I$ is finite dimensional and so has a faithful (finite-dimensional) representation $\tau$, say in $V$. It follows from Lemma 1 that $I \cap K=0$. Hence the composite mapping

$$
K \rightarrow U K \rightarrow(U K) / I \stackrel{\tau}{\rightarrow} \text { End } V
$$

gives a faithful representation $\sigma$ of $K$ in $V$, and $\sigma(K)^{q+1}=0$ since $K^{q+1} \subseteq I$.

We now use Proposition 7.3.1 of [Bo] to obtain an aggrandizement $\rho$ of $\sigma$ to $L$ (that is, a representation $\rho$ of $L$, say in $V^{\prime}$, for which there exists an injective $K$-module map of $V$ into $V^{\prime}$ ) which is nilpotent on $N$. The proof in [Bo] obtains $\rho$ by a chain of aggrandizements, where at each step the result follows from the aggrandizement theorem of Zassenhaus (Theorem 7.2.1 of [Bo]). We shall now observe that in each of these applications of Zassenhaus' theorem, the aggrandizement annihilates $K^{q+1}$. In each case we are dealing with a subalgebra $H=M+H^{\prime}$ of $L$ which is a vector space direct sum of a subalgebra $M$ and an ideal $H^{\prime}$ of $H$ with $K \subseteq H^{\prime}$, and a representation $\rho^{\prime}$ of $H^{\prime}$ (acting say in $W$ ) having the desired nilpotency properties. To obtain an aggrandizement of $\rho^{\prime}$ to $H$, one starts with the sub- $H$-module $S$ of $\left(U H^{\prime}\right)^{*}$ generated by the space

$$
C\left(\rho^{\prime}\right)=\left\{\lambda \circ \rho^{\prime} \mid \lambda \in(\text { End } W)^{*}\right\},
$$

of representative functions of $U H^{\prime}$ corresponding to the representation $\rho^{\prime}$, where if $y \in M$, then $y$ acts on $\left(U H^{\prime}\right)^{*}$ via $y f(u)=f(u y-y u)$ and if $y \in H^{\prime}$ then $y$ acts via $y f(u)=f(u y)$. In [Bo] it is shown that for some $n, S \oplus \cdots \oplus S$ ( $n$ times) furnishes the desired aggrandizement. In our case it thus suffices to show that $K^{q+1} S=0$. Since $\rho^{\prime}(K)^{q+1}=0$, we have $K^{q+1} C\left(\rho^{\prime}\right)=0$. Also $K^{q+1}(U M) \subseteq(U M) K^{q+1}$. Therefore

$$
\begin{aligned}
K^{q+1} S & =K^{q+1}(U M)\left(U H^{\prime}\right) C\left(\rho^{\prime}\right) \\
& =K^{q+1}(U M) C\left(\rho^{\prime}\right) \subseteq(U M) K^{q+1} C\left(\rho^{\prime}\right)=0 .
\end{aligned}
$$

Thus the aggrandizement of $\sigma$ to $L$ is a representation which is nilpotent on $N$, annihilates $K^{q+1}$, and is faithful on $K$ (since $\sigma$ is faithful). Adding to this representation the adjoint representation on $L$ (which also annihilates $K^{q+1}$ ), we get the desired faithful (since $z L \subseteq K$ ) representation of $L$. This completes the proof of Theorem 1 at characteristic 0 .

We now prove Theorem 2 at characteristic 0 . Let $y_{1}, \ldots, y_{n}$ be a basis of $L$ and $b_{1}, \ldots, b_{n}$ the dual basis of $L^{*}$. Let $\rho$ (acting in $V$ ) be a representation of 
$L$ satisfying the conclusion of Theorem 1 . If we regard $L$ as embedded (by $\rho$ ) in End $V$, then any linear functional on $L$ can be extended to a linear functional on End $V$. Hence for $i=1, \ldots, n$, there exists $\lambda_{i} \in(\text { End } V)^{*}$ such that $\lambda_{i} \circ \rho=b_{i}$ (on $L$ ). We write $\lambda_{i} \circ \rho=f_{i}$, regarded as a linear functional on $U L$. By taking (if necessary) a direct sum of $\rho$ with a trivial representation, we may assume that $f_{i}(1)=0, i=1, \ldots, n$.

The $f_{i}$ are elements of $R(L)$, the space of representative functions of $U L(R(L)$ is the sum, over all representations $\pi$, of the corresponding space $C(\pi)$ of functions for the representation $\pi ; R(L)$ is also denoted $(U L)^{0}$ - see [Sw]). We recall [Ho] that $R(L)$ is a commutative algebra (in fact a Hopf algebra) with multiplication given by

$$
\left(\lambda_{1} \circ \pi_{1}\right)\left(\lambda_{2} \circ \pi_{2}\right)=\left(\lambda_{1} \otimes \lambda_{2}\right) \circ\left(\pi_{1} \otimes \pi_{2}\right),
$$

and unit element $\varepsilon$ given by $\varepsilon(1)=1, \varepsilon(L \cdots L)=0$ ( $i$ factors of $L)$ for $i \geq 1$. For $f \in R(L)$, we write $\gamma_{j} f$ for the divided power $f^{j} / j$ !.

We use the basis of $U L$ consisting of the standard monomials

$$
\left\{y_{1}^{t_{1}} \cdots y_{n}^{t_{n}} \mid t_{1}, \ldots, t_{n} \geq 0\right\} .
$$

Since $f_{i}\left(y_{i}\right)=\delta_{i j}$ and $f_{i}(1)=0$, it follows from the formula for the tensor product of representations of $U L$ that

$$
\left(\left(\gamma_{s_{1}} f_{1}\right) \cdots\left(\gamma_{s_{n}} f_{n}\right)\right)\left(y_{1}^{s_{1}} \cdots y_{n}^{s_{n}}\right)=1
$$

and that $\left(\gamma_{s_{1}} f_{1}\right) \cdots\left(\gamma_{s_{n}} f_{n}\right)$ annihilates all other standard monomials of degree $\leq s_{1}+\cdots+s_{n}$. Suppose $r>0$ and $x \in(U L)_{r}-(U L)_{r-1}$. Then

$$
\left(\gamma_{s_{1}} f_{1}\right) \cdots\left(\gamma_{s_{n}} f_{n}\right) x \neq 0
$$

for some $s_{1}, \ldots, s_{n}$ with $s_{1}+\cdots+s_{n}=r$. We write $\pi=\otimes^{r} \rho(=\rho \otimes \cdots \otimes \rho(r$ times)). Then

$$
\left(\gamma_{s_{1}} f_{1}\right) \cdots\left(\gamma_{s_{n}} f_{n}\right) x=\left(\left(1 / s_{1} ! \cdots s_{n} !\right)\left(\left(\bigotimes^{s_{1}} \lambda_{1}\right) \otimes \cdots \otimes\left(\bigotimes^{s_{n}} \lambda_{n}\right)\right) \circ \pi\right) x
$$

Therefore $\pi x \neq 0$. Hence $(\operatorname{ker} \pi) \cap(U L)_{r} \subseteq(U L)_{r-1}$. Since $\rho\left(K^{q+1}\right)=0$, we have $\pi\left(K^{q r+1}\right)=0$. Since $\rho$ is nilpotent on $N$ so is $\pi$. By induction on $r$ (for $r=0$ one can use a trivial representation), there is a representation $\pi^{\prime}$ of $L$ faithful on $(U L)_{r-1}$, with $\pi^{\prime}(N)$ nilpotent and $\pi^{\prime}\left(K^{q(r-1)+1}\right)=0$. Hence $\pi^{\prime}\left(K^{q+1}\right)=0$, and $\pi \oplus \pi^{\prime}$ has the required properties. This completes the proof of Theorem 2 at characteristic 0 .

REMARK. The above proof actually shows the following. Let $\rho$ be a faithful representation of $L$, and define $\rho^{\prime}$ to be either $\rho$ or the sum of $\rho$ and the trivial representation accordingly as $\rho(L)$ does not or does contain the identity. Then

$$
\rho^{\prime} \oplus\left(\rho^{\prime} \otimes \rho^{\prime}\right) \oplus \cdots \oplus\left(\bigotimes^{r} \rho^{\prime}\right)
$$

is faithful ${ }^{3}$ on $(U L)_{r}$ (and annihilates $K^{q r+1}$ if $\rho$ annihilates $K^{q+1}$ ).

Our representation has been chosen so that it is also nilpotent on $N$. By a result of Hochschild [Ho2], this has the following consequence.

\footnotetext{
${ }^{3}$ The proof of this can be extended to prime characteristic by using the divided powers in $R(L)$ in that case. For the existence of these divided powers, see [BI].
} 
COROLLARY 1. At characteristic 0 there exists a representation as in Theorem 2 with the additional property that it is nilpotent on each $x \in L$ for which ad $x$ is nilpotent.

We now prove Theorem 2 in the characteristic $p$ case. We extend the basis $\left\{x_{1}, \ldots, x_{s}\right\}$ of $K$ to a basis $\left\{x_{1}, \ldots, x_{n}\right\}$ of $L$ By Lemma 6.5 of $[\mathbf{J a 2}]$, for each $i$ there exists a $p$-polynomial $m_{i}(\lambda)$, of degree $d_{i}$, such that $z_{i}=m_{i}\left(x_{i}\right)$ is in the center of $U L$. We may assume that $d_{i}>r$. We write

$$
B=\left\{z_{s+1}^{h_{s+1}} \cdots z_{n}^{h_{n}} x_{1}^{j_{i}} \cdots x_{n}^{j_{n}} \mid h_{i}, j_{i} \geq 0, j_{l}<d_{l}(l>s)\right\}
$$

Then $B$ is a basis of $U L$ (cf. Lemma 5.4 of [Ja2]). For $b \in B$ we denote the corresponding $h_{i}$ (with $i>s$ ) by $h_{i}(b)$ and $\sum_{i=1}^{s} j_{i} w\left(x_{i}\right)$ by $w(b)$. We set

$$
B^{\prime}=\left\{b \in B \mid w(b)>q r \text { for some } h_{i}(b)>0\right\},
$$

and let $J$ be the ideal of $U L$ generated by $z_{s+1}, \ldots, z_{n}$ and $K^{q r+1}$. Then

$$
J=(U L) K^{q r+1}(U L)+\sum_{i>s} z_{i}(U L)=K^{q r+1}(U L)+\sum_{i>s} z_{i}(U L) .
$$

Also $B^{\prime} \subseteq J$, and $K^{q r+1}$ is in the span of $B^{\prime}$, by Lemma 1 . If $b \in B$ then $z_{i} b \in B^{\prime}$ for $i>s$, and $K^{q r+1} b$ is in the span of the elements $y$ of $B^{\prime}$ with $w(y)>q r$, by Lemma 1. It follows that $z_{i}(U L)$ and $K^{q r+1}(U L)$ are contained in the span of $B^{\prime}$. Therefore $B^{\prime}$ is a basis of $J$, the cosets of the elements of $B-B^{\prime}$ form a basis of $(U L) / J$, and $(U L) / J$ is finite dimensional. The standard monomials (in $x_{1}, \ldots, x_{n}$ ) of degree $\leq r$ form a basis of $(U L)_{r}$. Every such monomial is in $B-B^{\prime}$ since each $d_{i}>r(i>s)$ and since $w\left(x_{i}\right) \leq q(i \leq s)$. Hence the map $U L \rightarrow(U L) / J$ is faithful on $(U L)_{r}$. Composing this with a faithful representation of $(U L) / J$, we get a representation $\rho$ of $U L$ which is faithful on $(U L)_{r}$. Moreover $\rho(K)^{q r+1}=0$.

The following is a sharpened version, for algebras containing a nonzero nilpotent ideal, that is, for nonsemisimple algebras, of the existence of indecomposable representations of arbitrarily high degree.

COROLlary 2. Suppose $K$ is an ideal of $L, K$ is nilpotent of class $q, 0 \neq x \in$ $K$, and $r>0$. Then there exists an indecomposable representation $\sigma$ of $L$ such that $\sigma(x)^{r} \neq 0$ but $\sigma(x)^{q r+1}=0$.

\section{REFERENCES}

[Bl] R. E. Block, Commutative Hopf algebras, Lie coalgebras, and divided powers J. Algebra 96 (1985), 275-306.

[Bo] N. Bourbaki, Groupes et algèbres de Lie, Chap. I (2nd ed.), Actualités Sci. Indust., no. 1285, Hermann, Paris, 1971.

[Di] J. Dixmier, Algèbres enveloppantes, Gauthier-Villars, Paris, 1974; English transl., Enveloping algebras, North-Holland, Amsterdam, 1977.

[Ha] Harish-Chandra, On representations of Lie algebras, Ann. of Math. 50 (1949), 900-915.

[Ho] G. Hochschild, Algebraic Lie algebras and representative functions, Illinois J. Math 3 (1959), 499523.

[Ho2] _ An addition to Ado's theorem, Proc. Amer. Math. Soc. 17 (1966), 531-533.

[Ja] N. Jacobson, A note on Lie algebras of characteristic p, Amer. J. Math. 74 (1952), 357-359.

[Ja2] _ Lie algebras, Wiley-Interscience, New York, 1962.

[Sw] M. E. Sweedler, Hopf algebras, Benjamin, New York, 1969.

Department of Mathematics, University of CAlifornia, Riverside, CaliforNIA 92521 